\title{
MicroRNA-519a promotes tumor growth by targeting PTEN/PI3K/AKT signaling in hepatocellular carcinoma
}

\author{
KANGSHENG TU, ZHIKUI LIU, BOWEN YAO, SHAOSHAN HAN and WEI YANG \\ Department of Hepatobiliary Surgery, the First Affiliated Hospital \\ of Xi'an Jiaotong University, Xi'an, Shaanxi 710061, P.R. China
}

Received November 24, 2015; Accepted December 8, 2015

DOI: $10.3892 /$ ijo.2015.3309

\begin{abstract}
MicroRNAs (miRNAs) have been found to play fundamental roles in the pathogenesis of hepatocellular carcinoma (HCC). Previous miRNA array data showed that miR-519a was upregulated in HCC tissues compared to adjacent non-tumor tissues. However, the functional role of miR-519a in HCC remains unexplored. In this study, we demonstrated that the expression of miR-519a was elevated in both HCC tissues and cell lines. Clinical association analysis revealed that high expression of miR-519a was correlated with adverse clinicopathological characteristics including large tumor size, high Edmondson-Steiner grading, advanced tumor-node-metastasis (TNM) tumor stage and venous infiltration. Furthermore, high expression of miR-519a conferred a reduced 5-year overall survival and disease-free survival of HCC patients. Moreover, we disclosed that miR-519a overexpression promoted, but miR-519a silencing reduced, HCC cell proliferation and cell cycle progression in vitro. Notably, we identified phosphatase and tensin homolog (PTEN) as a direct downstream target and functional mediator of miR-519a in HCC cells. Mechanistically, phosphatidylinositol-3-OH kinase (PI3K)/AKT pathway, downstream of PTEN, is essential for the functional roles of miR-519a in HCC cells. In conclusion, our results indicate that miR-519a promotes tumor growth of HCC by targeting PTENmediated PI3K/AKT pathway, and potentially serves as a novel prognostic biomarker and therapeutic target for HCC.
\end{abstract}

\section{Introduction}

Hepatocellular carcinoma (HCC) is one of the most common cancers worldwide and the third leading causes of cancer-

Correspondence to: Professor Wei Yang, Department of Hepatobiliary Surgery, the First Affiliated Hospital of Xi'an Jiaotong University, No. 277 Yanta West Road, Xi'an, Shaanxi 710061, P.R. China E-mail: dr_bobyang@163.com

Key words: microRNA-519a, hepatocellular carcinoma, phosphatase and tensin homolog, PI3K/AKT signaling, tumor growth related death $(1,2)$. Despite recent advances in clinical and experimental treatment, the prognosis of HCC remains poor with a dismal 5-year survival (3), and the detailed mechanism underlying the development and progression of HCC has not been fully elucidated (4). Therefore, it is of great importance to clarify the molecular mechanisms of HCC and develop novel strategies for the diagnosis and treatment of HCC.

MicroRNAs (miRNAs) are endogenous non-coding 20-22 nucleotide RNAs that regulate protein expression by interacting with complementary sites within the 3'-untranslated region (UTR) of target mRNAs (5-7). Increasing evidence suggests that aberrant expression of miRNAs plays crucial roles in various cellular processes, including cell proliferation, apoptosis, differentiation, migration and invasion (8-13). In addition, it has been widely recognized that deregulation of miRNAs may lead to abnormal expression of oncogenes or tumor suppressors and contribute to the development and progression of human cancers.

miR-519a, which belongs to a large cluster of miRNAs, C19MC, plays a critical role in the pathogenesis of human cancers. It played an oncogenic role and could serve as a diagnostic and prognostic biomarker in ovarian epithelial tumors (14). Moreover, it was recently found to confer tamoxifen resistance by targeting the phosphatase and tensin homolog (PTEN), retinoblastoma protein (RB1), and cyclindependent kinase inhibitor $1 \mathrm{~A}(\mathrm{CDKN} 1 \mathrm{~A}) / \mathrm{p} 21$ in estrogen receptor $(E R)^{+}$breast cancer (15). Studies have shown that miR-519a functioned as a tumor suppressor through multiple p21-inducing pathways in HeLa human cervical carcinoma (16). Moreover, miR-519a reduced cell proliferation by lowering RNA-binding protein HuR levels in colon carcinoma (17). Thus, the exact roles of miR-519a in human cancers are cancer-type specific. However, the functional role of miR-519a and the underlying mechanisms in HCC are still unknown.

In this study, we demonstrated that upregulation of miR-519a was associated with poor prognostic features and reduced 5-year survival of HCC patients. Gain- and lossof-function studies revealed that miR-519a promoted HCC cell proliferation and cell cycle progression. Finally, we identified PTEN and PI3K/AKT pathway as direct targets of miR-519a. 


\section{Materials and methods}

Clinical tissues and data. Tumor samples and matched tumoradjacent tissues were obtained from 116 patients undergoing curative resection of their primary HCC in the Department of Hepatobiliary Surgery at the First Affiliated Hospital of Xi'an Jiaotong University during January 2006 to December 2009. Clinical sample was used after obtaining informed consent from each patient. None of the patients had received any perioperative chemo- or radiotherapy. The demographic and clinicopathological data of all enrolled patients were obtained through review of hospital records, and are presented in Table I. The protocols of this study were approved by the Xi'an Jiaotong University Ethics Committee according to the Declaration of Helsinki.

Cell culture and treatment. The human immortalized normal hepatic cell line LO2 and HCC cell lines (HepG2, Hep3B, Huh7, SMMC-7721 and Bel-7402) were obtained from the Chinese Academy of Sciences (Shanghai, China). The cells were cultured in Dulbecco's modified Eagle's medium (DMEM; Invitrogen, Carlsbad, CA, USA) supplemented with $10 \%$ fetal bovine serum (Gibco, Grand Island, NY, USA) at $37^{\circ} \mathrm{C}$ with $5 \% \mathrm{CO}_{2}$. Akt inhibitor MK-2206 $(1 \mu \mathrm{M}$, Selleck Chemicals, Houston, TX, USA) was used to treat HCC cells following the manufacturer's instructions.

Quantitative real-time polymerase chain reaction ( $q R T-P C R)$. Total RNA was extracted from clinical specimens or HCC cells using TRIzol reagent (Invitrogen) following manufacturer's instruction. PCR amplification was performed using a TaqMan human miRNA assay kit (Applied Biosystems, Foster City, CA, USA) and a SYBR ${ }^{\circledR}$ Premix Ex Taq $^{\mathrm{TM}}$ II (Perfect Real-Time) kit (Takara Bio Inc., Shiga, Japan) with an ABI PRISM 7300 Sequence Detection System (Applied Biosystems). qPCR primer against mature miRNA hsamiR-519a-3p (HmiRQP0588), Homo sapiens snRNA U6 qPCR Primer (HmiRQP9001), PTEN (HQP015535) and GAPDH (HQP006940) were purchased from Genecopoeia (Guangzhou, China).

Western blot analysis. Total protein was extracted from HCC cells and $40 \mu \mathrm{g}$ of isolated protein was separated by $10 \%$ SDS-PAGE and transferred onto a PVDF membrane (BioRad Laboratories, Hercules, CA, USA). The membranes were probed with the following primary antibodies: Akt (1:1000, Cell Signaling Technology, Inc., Danvers, MA, USA), p-Akt (1:1000, Cell Signaling Technology, Inc.), PTEN (1:1000, Cell Signaling Technology, Inc.), Cyclin D (1:1000, Cell Signaling Technology, Inc.), and p27 (1:1000, Cell Signaling Technology, Inc.) overnight. Then the membranes were incubated with the HRP-conjugated goat anti-mouse or anti-rabbit IgG antibody (ZSGB-BIO, China). Protein bands were visualized using an enhanced chemiluminescence kit (Amersham, Little Chalfont, UK).

Immunohistochemical staining. Immunohistochemistry was performed on paraformaldehyde-fixed paraffin sections. PTEN (1:100, Cell Signaling Technology, Inc.) antibody was used in immunohistochemistry by a streptavidin
Table I. Correlation between the clinicopathological characteristics and miR-519a expression in HCC $(n=116)$.

\begin{tabular}{|c|c|c|c|c|}
\hline \multirow[b]{2}{*}{$\begin{array}{l}\text { Clinical } \\
\text { parameters }\end{array}$} & \multirow[b]{2}{*}{$\mathrm{n}$} & \multicolumn{2}{|c|}{ Expression level } & \multirow[b]{2}{*}{ P-value } \\
\hline & & $\begin{array}{l}\operatorname{miR}-519 a^{\text {high }} \\
\quad(\mathrm{n}=64)\end{array}$ & $\begin{array}{c}\operatorname{miR}-519 a^{\text {low }} \\
\quad(\mathrm{n}=52)\end{array}$ & \\
\hline \multicolumn{5}{|l|}{ Age (years) } \\
\hline$<65$ years & 61 & 34 & 27 & 0.897 \\
\hline$\geq 65$ years & 55 & 30 & 25 & \\
\hline \multicolumn{5}{|l|}{ Gender } \\
\hline Male & 89 & 49 & 40 & 0.964 \\
\hline Female & 27 & 15 & 12 & \\
\hline Tumor size (cm) & & & & $0.005^{\mathrm{a}}$ \\
\hline$<5$ & 78 & 36 & 42 & \\
\hline$\geq 5$ & 38 & 28 & 10 & \\
\hline Tumor number & & & & 0.972 \\
\hline Solitary & 98 & 54 & 44 & \\
\hline Multiple & 18 & 10 & 8 & \\
\hline \multicolumn{5}{|l|}{ Edmondson } \\
\hline I+II & 71 & 33 & 38 & $0.018^{\mathrm{a}}$ \\
\hline III+IV & 45 & 31 & 14 & \\
\hline TNM stage & & & & $0.013^{\mathrm{a}}$ \\
\hline $\mathrm{I}+\mathrm{II}$ & 93 & 46 & 47 & \\
\hline III+IV & 23 & 18 & 5 & \\
\hline Capsular infiltration & & & & 0.599 \\
\hline Present & 70 & 40 & 30 & \\
\hline Absent & 46 & 24 & 22 & \\
\hline Venous infiltration & & & & $0.005^{\mathrm{a}}$ \\
\hline Present & 19 & 16 & 3 & \\
\hline Absent & 97 & 48 & 49 & \\
\hline $\operatorname{AFP}(\mathrm{ng} / \mathrm{ml})$ & & & & 0.947 \\
\hline$<400$ & 42 & 23 & 19 & \\
\hline$\geq 400$ & 74 & 41 & 33 & \\
\hline HBV & & & & 0.873 \\
\hline Positive & 110 & 60 & 50 & \\
\hline Negative & 6 & 4 & 2 & \\
\hline
\end{tabular}

HCC, hepatocellular carcinoma; HBV, hepatitis B virus; AFP,

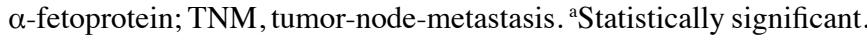

peroxidase-conjugated (SP-IHC) method. Detailed procedure of immunohistochemistry was performed as previously reported (18).

Plasmids and cell transfection. miRNA vectors, including miR-519a expression vector (HmiR0342-MR04), the control vector for miR-519a (CmiR0001-MR04, miR-control), miR-519a inhibitor (HmiR-AN0588-AM04, anti-miR-519a) and the negative control for the miR-519a inhibitor (CmiRAN0001-AM04, anti-miR-NC), and PTEN expression plasmid (EX-I0450-M02-5) were purchased from Genecopoeia 

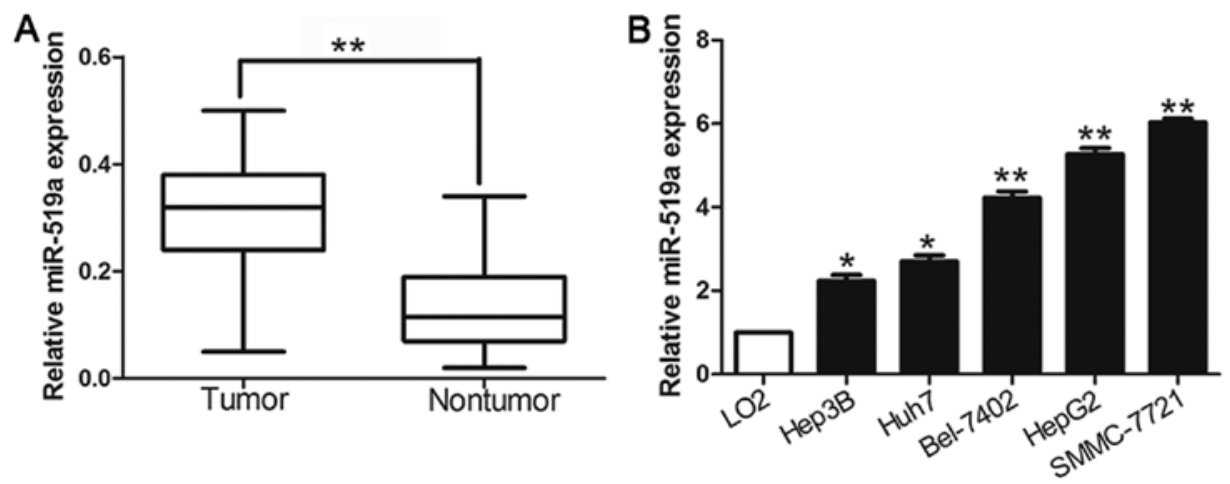

Figure 1. The expression of miR-519a is significantly elevated in HCC. (A) Relative miR-519a expression levels in HCC tissues and matched adjacent normal tissues were determined by qRT-PCR ( $\mathrm{n}=116)$. (B) The expression of miR-519a in five HCC cell lines was significantly increased compared with that in the LO2 cells. U6 snRNA was used as internal control. ${ }^{*} \mathrm{P}<0.05,{ }^{* *} \mathrm{P}<0.01$.
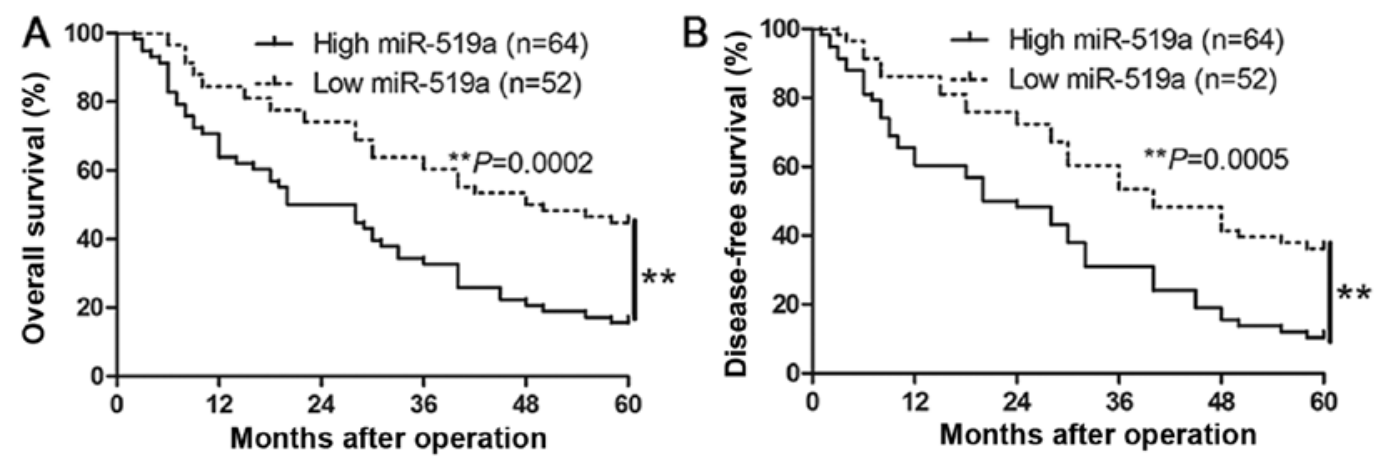

Figure 2. The prognostic value of miR-519a for HCC patients. HCC patients with higher expression of miR-519a had worse (A) overall survival and (B) disease-free survival. ${ }^{* *} \mathrm{P}<0.01$.

(Guangzhou, China). The PTEN siRNA duplex sequence, 5'-GUU AGC AGA AAC AAA AGG AGA UAU CAA-3' (sense)/5'-UUG AUA UCU CCU UUU GUU UCU GCU AAC-3' (antisense) and a nonspecific duplex oligonucleotide as a negative control were synthesized by Sangon Biotech Co., Ltd. (Shanghai, China). Cells were transfected with oligonucleotides using Lipofectamine 2000 Reagent (Invitrogen Life Technologies) following the manufacturer's instructions.

Luciferase assay. Cells were seeded in triplicate in 24-well plate and allowed to settle for $\sim 12 \mathrm{~h}$. pGL3-PTEN was co-transfected into HCC cells with TK-Renilla plasmid as control signals using Lipofectamine 2000. Luciferase and control signals were measured at $48 \mathrm{~h}$ after transfection using the Dual Luciferase Reporter Assay kit (Promega, Madison, WI, USA), according to a protocol provided by the manufacturer. Three independent experiments were performed and the data are presented as the mean \pm SD.

MTT and colony formation assays. At $48 \mathrm{~h}$ after transfection, $5 \times 10^{3}$ cells were seeded into 96-well plates and stained with $0.5 \mathrm{mg} / \mathrm{ml}$ sterile MTT (Sigma-Aldrich, St. Louis, MO, USA) for $4 \mathrm{~h}$ at $37^{\circ} \mathrm{C}$. Then the culture medium was discarded and an extra $150 \mu \mathrm{l}$ DMSO (Sigma-Aldrich) were added. The absorbance at $490 \mathrm{~nm}$ was measured at 24,48 and $72 \mathrm{~h}$ after transfection. The colony formation assay was performed as previously described. Briefly, 500 cells per well were seeded on six-well plates. After 2 weeks, the colonies were stained with $1 \%$ crystal violet and the number of colonies was counted.

Cell cycle and cell proliferation assays. At $48 \mathrm{~h}$ after transfection, HCC cells were collected for cell cycle analysis. After being washed with PBS three times, the cells were fixed with $80 \%$ ethanol overnight at $-20^{\circ} \mathrm{C}$, and were subsequently treated with RNaseA (Sigma) for $30 \mathrm{~min}$ at $37^{\circ} \mathrm{C}$, followed by incubation in $20 \mu \mathrm{g} / \mathrm{ml}$ of propidium iodide (Sigma) for $20 \mathrm{~min}$ at room temperature. After incubation, the cells were subjected to flow cytometry analysis using a FACS Calibur (BD Biosciences, Bedford, MA, USA). For proliferation, 5-bromodeoxyuridine (BrdU) labeling and immunofluorescence was used. Cells grown on cover slips (Fisher Scientific, Pittsburgh, PA, USA) were incubated with BrdU for $1 \mathrm{~h}$ and stained with anti-BrdU antibody (Sigma) according to the manufacturer's instruction. Gray level images were acquired under a laser scanning microscope (Axioskop 2 plus, Carl Zeiss Co. Ltd., Jena, Germany).

Statistical analysis. Data are presented as the mean \pm SD from at least three independent replicates. SPSS software, 16.0 (SPSS, Inc, Chicago, IL, USA) was used to conduct the analysis, and a two-tailed Student t-test was employed to analyze the differences between two groups. Pearson's correlation analysis was used to analyze the correlation between two indices. Survival curves were plotted by the Kaplan-Meier 

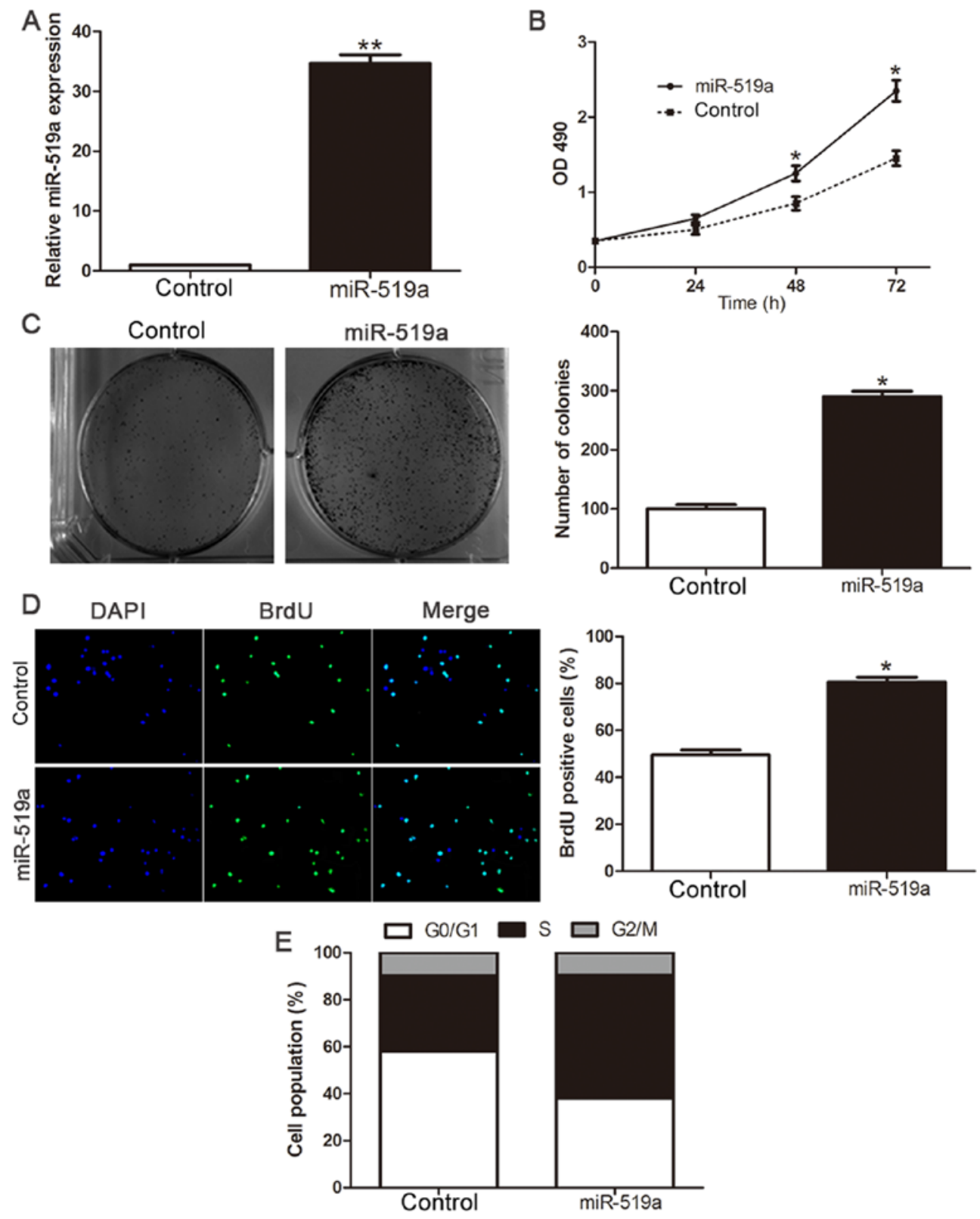

Figure 3. Overexpression of miR-519a promotes the growth of Hep3B cells. (A) The expression of miR-519a was measured in Hep3B cells transfected with miR-519a mimics or control vector. (B) Cell viability was evaluated by the MTT assay at the indicated days. (C) Representative results for colony formation by the indicated cells. (D) Representative micrographs (left) and quantification (right) of BrdU-incorporating cells of indicated Hep3B cells. (E) Effects of miR-519a overexpression on the cell cycle progression of Hep3B cells measured by flow cytometric analysis. Experiments were repeated at least 3 times with similar results, and error bars represent $\pm \mathrm{SD}$. ${ }^{*} \mathrm{P}<0.05$.

method and compared using the log-rank test. Differences were considered statistically significant at $\mathrm{P}<0.05$.

\section{Results}

Expression of miR-519a is upregulated in HCC. We investigated the expression level of miR-519a in 116 pairs of HCC tissues and matched tumor-adjacent tissues using qRT-PCR. The results showed that the mean level of miR-519a expression in HCC tissues was significantly higher than that in the non-tumor tissues $(\mathrm{P}<0.05$, Fig. 1A). Further results confirmed that miR-519a was upregulated in a panel of HCC cell lines (HepG2, Huh7, Hep3B, SMMC-7721 and Bel-7402) compared with that in non-transformed $\mathrm{LO} 2$ hepatic cell line $(\mathrm{P}<0.05$,
Fig. 1B). The results suggest that elevated expression of miR519a may contribute to the development of HCC.

Clinical significance of miR-519a expression in HCC specimens. We determined the mean level of miR-519a as a cut-off value to investigate the correlation between miR-519a level and the clinical features and prognosis of HCC patients. As shown in Table I, the high expression of miR-519a was prominently associated with large tumor size $(\mathrm{P}=0.005)$, high Edmondson-Steiner grading $(\mathrm{P}=0.018)$, advanced tumornode-metastasis $(\mathrm{TNM})$ tumor stage $(\mathrm{P}=0.013)$ and venous infiltration $(\mathrm{P}=0.005)$. Furthermore, Kaplan-Meier analysis showed that high miR-519a expression was closely associated with shorter overall survival $(\mathrm{P}=0.0002$, Fig. $2 \mathrm{~A})$ and 


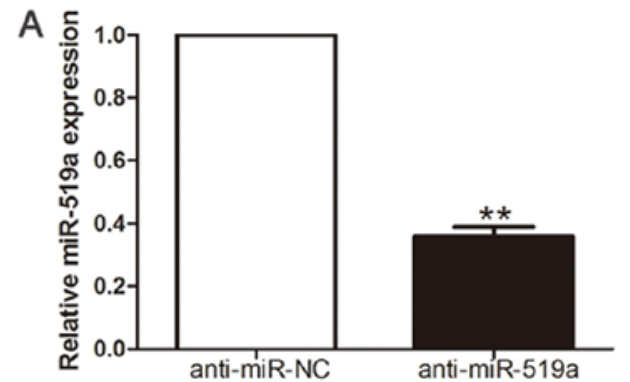

B
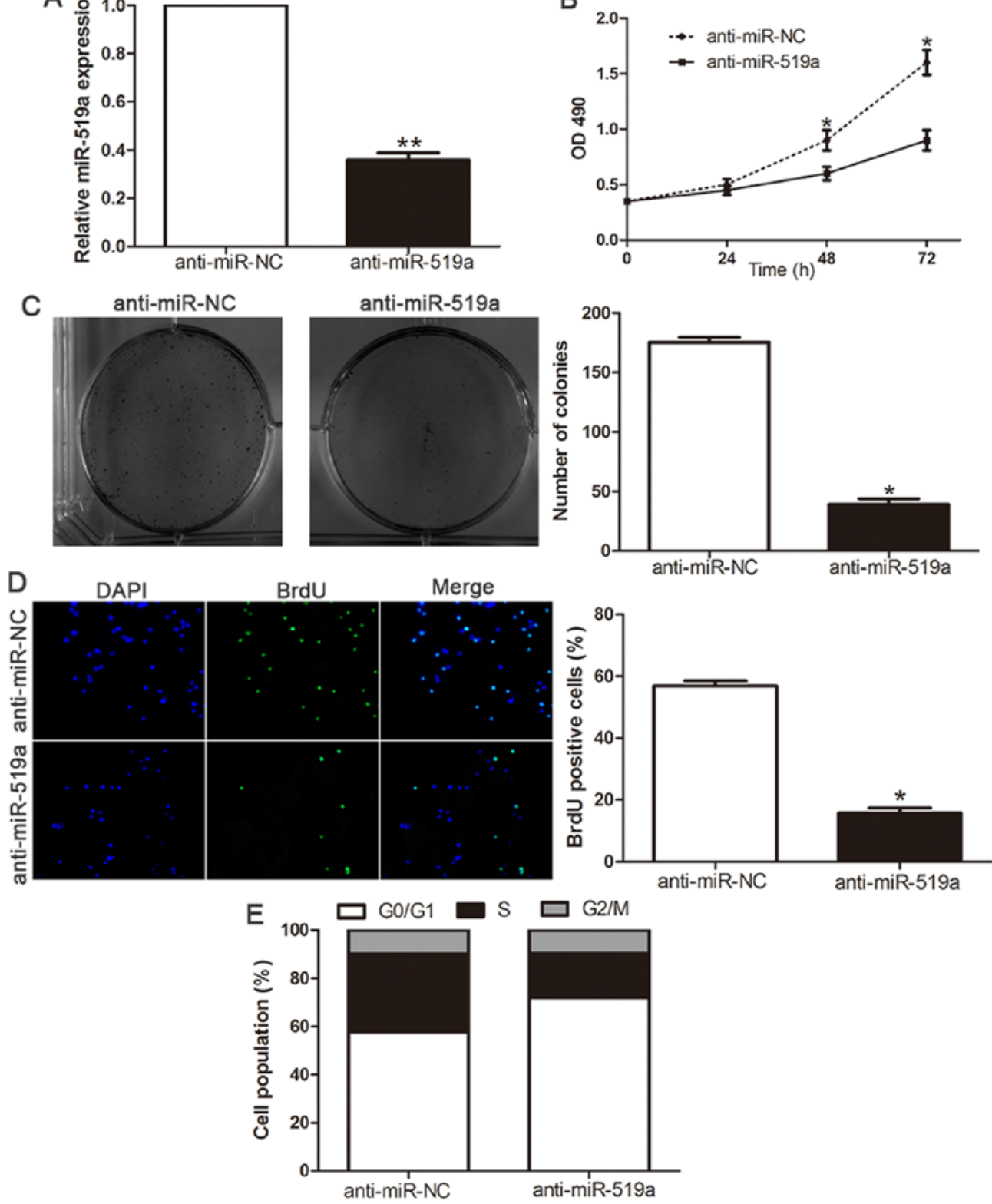

Figure 4. Downregulation of miR-519a inhibits the growth of SMMC-7721 cells. (A) The expression of miR-519a was measured in SMMC-7721 cells transfected with miR-519a inhibitor or scramble vector. (B) Cell viability was evaluated by the MTT assay at the indicated days. (C) Representative results for colony formation by the indicated cells. (D) Representative micrographs (left) and quantification (right) of BrdU-incorporating cells of indicated SMMC-7721 cells. (E) Effects of miR-519a downregulation on the cell cycle progression of SMMC-7721 cells measured by flow cytometric analysis. Experiments were repeated at least 3 times with similar results, and error bars represent $\pm \mathrm{SD}$. ${ }^{*} \mathrm{P}<0.05$.

disease-free survival ( $\mathrm{P}=0.0005$, Fig. $2 \mathrm{~B})$, which highlights the potential value of miR-519a as a predictive biomarker for the outcome of HCC.

Overexpression of $\mathrm{miR}-519$ a promotes proliferation and cell cycle progression of HCC cells. To investigate the biological function of miR-519a in the development and progression of HCC, we transfected HCC cell line Hep3B with miR-519a mimic $(\mathrm{P}<0.01$, Fig. 3A). Cell viability was measured using MTT assays and we observed that ectopic expression of miR519a had more viability over time compared with NC group $(\mathrm{P}<0.05$, Fig. 3B). Consistently, the upregulated expression of miR-519a markedly enhanced the colony formation as suggested by the increase in colony number $(\mathrm{P}<0.05$, Fig. $3 \mathrm{C})$. Furthermore, the level of DNA synthesis, examined with
BrdU incorporation assay, was significantly elevated in miR519a transduced Hep3B cells, whereas the vector control cells displayed relatively lower BrdU incorporation rates $(\mathrm{P}<0.05$, Fig. 3D). Cell cycle analysis revealed that overexpression of miR-519a decreased the number of Hep3B cells in G0/G1 phase while increased the proportion of Hep3B cells in S-phase $(\mathrm{P}<0.05$, Fig. 3E). Collectively, these results demonstrate that miR-519a functions to enhance proliferation, tumorigenicity and cell cycle progression of HCC cells.

Inhibition of miR-519a attenuates proliferation and cell cycle progression of HCC cells. Loss-of-function studies were further performed to confirm the biological function by antimiR-519a vector. The expression of miR-519a was significantly decreased in SMMC-7721 cells $(\mathrm{P}<0.01$, Fig. 4A) following 
A

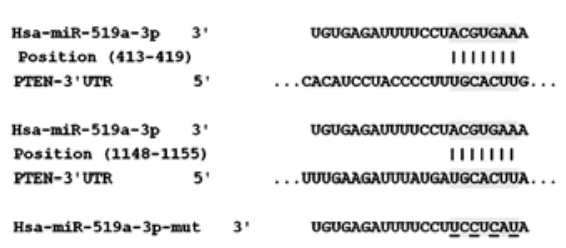

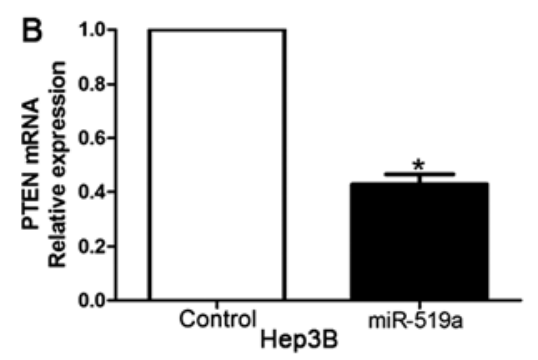
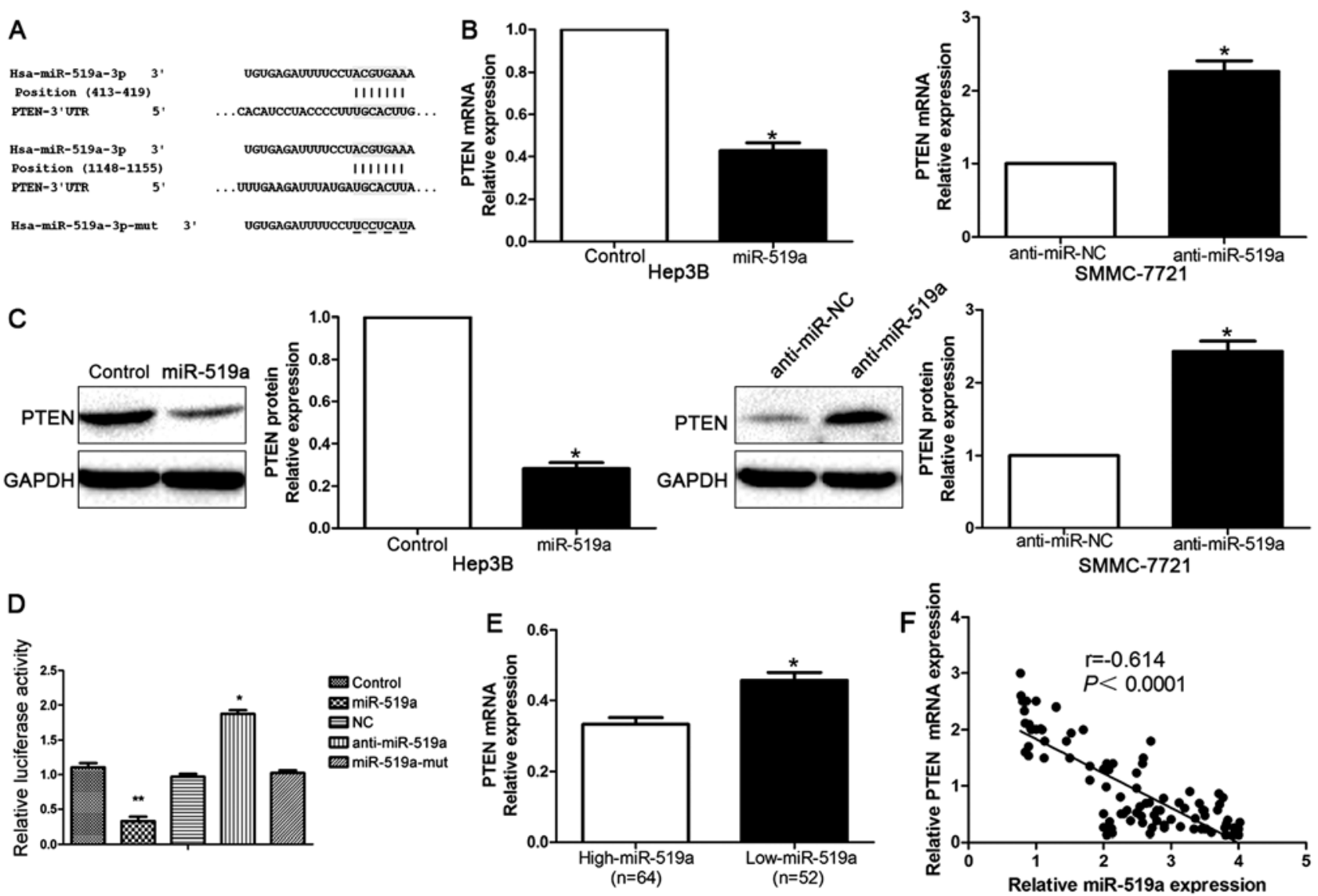

Figure 5. PTEN is a direct target of miR-519a in HCC cells. (A) The putative binding sequence of miR-519a in the 3'-UTR of PTEN. (B) qRT-PCR analysis of PTEN mRNA expression in Hep3B cells transfected with miR-519a mimics or control vector and in SMMC-7721 cells transfected with miR-519a inhibitor or negative control vector (NC); $\mathrm{n}=3$ repeats with similar results. (C) Overexpression of miR-519a reduced the expression of PTEN protein in Hep3B cells and knockdown of miR-519a increases the level of PTEN protein in SMMC-7721 cells; $n=3$ repeats with similar results. (D) miR-519a significantly suppressed the luciferase activity of PTEN. Anti-miR-519a led to a notable increase in the luciferase activity of 3'-UTR of PTEN. miR-519a-mut had no influence on the luciferase activity of PTEN; $n=3$ repeats with similar results. (E) The expression of PTEN mRNA in miR-519a high-expressing tumors was significantly lower than that in miR-519a low-expressing tumors. (F) A significant inverse correlation between the mRNA levels of miR-519a and PTEN was observed in HCC tissues. ${ }^{*} \mathrm{P}<0.05,{ }^{* *} \mathrm{P}<0.01$.

transfection of miR-519a inhibitors. As shown in Fig. 4B, downregulation of miR-519a led to a significant reduction of cell viability $(\mathrm{P}<0.05)$. Furthermore, suppression of miR-519a significantly inhibited the colony formation ability $(\mathrm{P}<0.05$, Fig. 4C). Moreover, BrdU incorporation assay confirmed that downregulation of miR-519a significantly inhibited the proliferation in SMMC-7721 cells $(\mathrm{P}<0.05$, Fig. 4D). In addition, flow cytometry showed inhibition of miR-519a in SMMC-7721 cells significantly increased the percentage of cells in G1/G0 phase and decreased the percentage of cells in $\mathrm{S}$ phase $(\mathrm{P}<0.05$, Fig. $4 \mathrm{E})$. These results demonstrated that miR-519a regulates the proliferation, tumorigenicity and cell cycle of HCC cells.

PTEN was a direct target of miR-519a in HCC. To further explore the underlying mechanisms by which miR-519a exerted its functional effects on HCC, we used TargetScan to search for potential downstream targets of miR-519a. As shown in Fig. 5A, we found that 3'-UTR of PTEN contained the highly conserved putative miR-519a binding sites. qRT-PCR and western blot analysis showed that ectopic expression of miR-519a markedly decreased, whereas inhibition of miR-519a increased the mRNA $(\mathrm{P}<0.05$, Fig. 5B) and
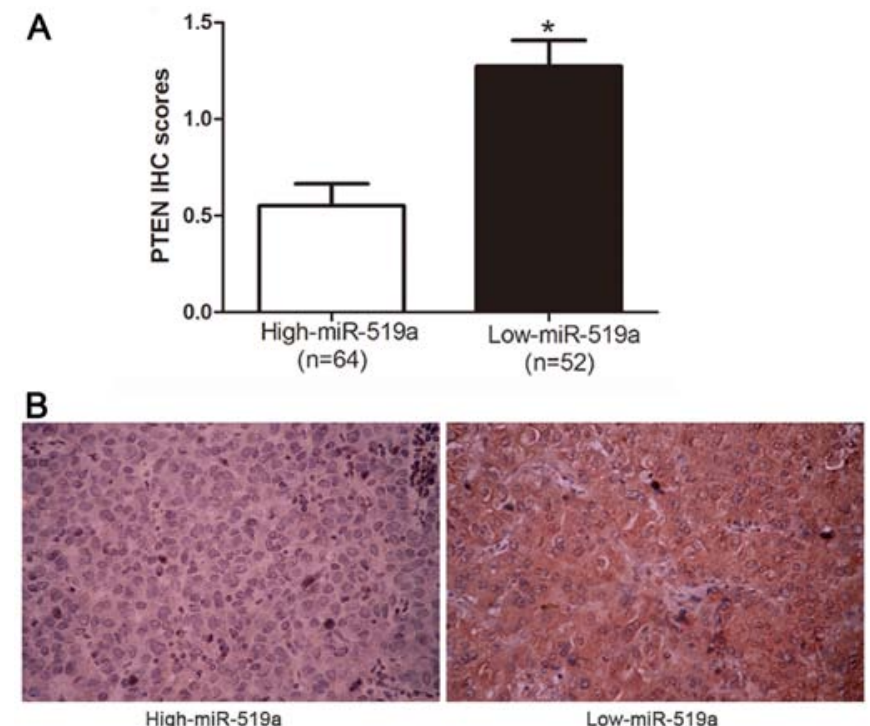

Figure 6. The expression of PTEN protein in HCC tissues. (A) The expression of PTEN protein in miR-519a high-expressing tumors was significantly lower than that in miR-519a low-expressing tumors. (B) Representative immunostaining showed negative expression of PTEN in miR-519a high-expressing $\mathrm{HCC}$ tissue and positive expression of PTEN in miR-519a low-expressing tumor. ${ }^{*} \mathrm{P}<0.05$. 
A
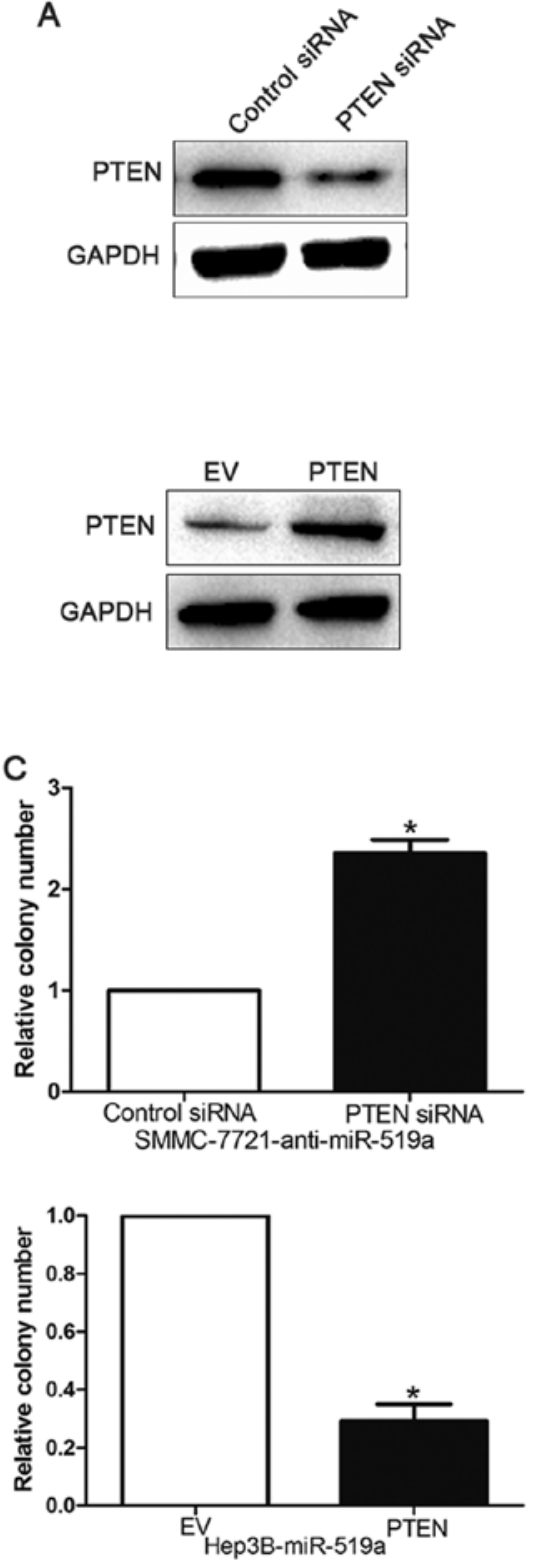
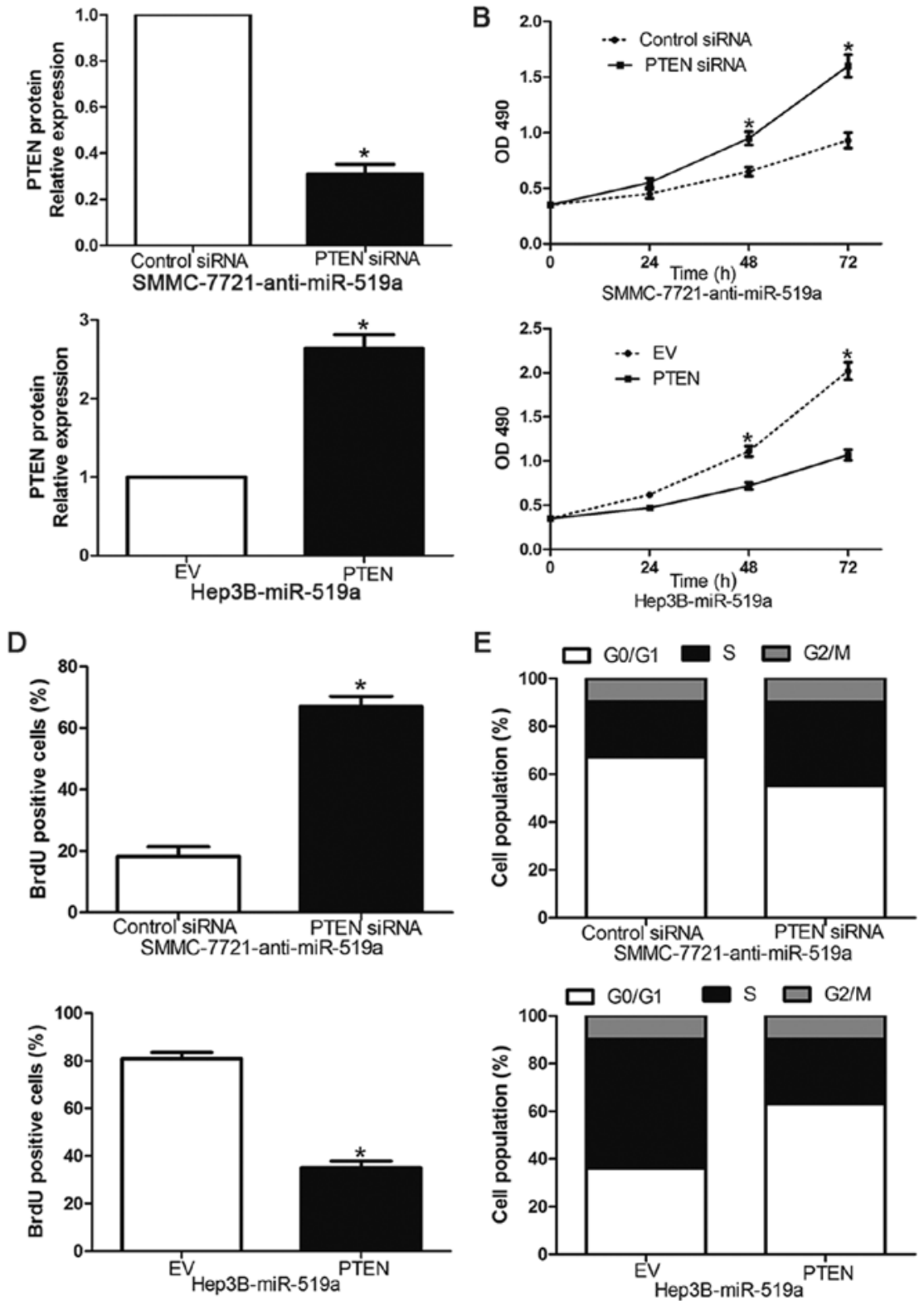

Figure 7. PTEN is the functional mediator downstream of miR-519a in HCC cells. (A) miR-519a-suppressive SMMC-7721 cells that were transfected with control siRNA or PTEN siRNA and miR-519a-overexpressing Hep3B cells that were transfected with EV or PTEN expression plasmid were subjected to western blot analysis for PTEN; $n=3$ repeats with similar results. Altering PTEN expression partly abolished the functional effect of miR-519a on (B) cell viability, (C) colony formation, (D) cell proliferation and (E) cell cycle progression; $\mathrm{n}=3$ independent experiments. ${ }^{*} \mathrm{P}<0.05{ }^{* * *} \mathrm{P}<0.01$.

protein $(\mathrm{P}<0.05$, Fig. 5C) expression of PTEN. Moreover, when co-transfected with PTEN-3' UTR luciferase reporter plasmid, as shown in Fig. 5D, miR-519a mimics led to significant reduction of luciferase activity of PTEN $(\mathrm{P}<0.01)$, while transfection of miR-519a inhibitors resulted in significant increase of luciferase activity of PTEN ( $\mathrm{P}<0.01$, Fig. 5D). In addition, the luciferase activity was unaffected by the miR519a-mut (Fig. 5D). To further validate that PTEN was a direct downstream target of miR-519a, we examined the correlation between miR-519a level and PTEN expression in HCC tissues. We found PTEN mRNA level in the miR-519a high-expressing tumors was significantly lower than those in the miR-519a low-expressing tumors $(\mathrm{P}<0.05$, Fig. 5E). Moreover, miR-519a level was inversely correlated with the level of PTEN mRNA in HCC tissues ( $\mathrm{r}=-0.614, \mathrm{P}<0.0001$, Fig. 5F). Consistently, as indicated by the IHC results, the protein expression of PTEN was obviously decreased in patients with high level of miR-519a $(\mathrm{P}<0.05$, Fig. 6). Collectively, these results strongly suggest that PTEN is a downstream target of miR-519a in HCC.

PTEN is a downstream mediator of the biological function of miR-519a in HCC. To evaluate whether PTEN could mediate the biological function of miR-519a in HCC, we inhibited PTEN expression with siRNAs in miR-519a-suppressive SMMC-7721 cells $(\mathrm{P}<0.05$, Fig. 7A). We found that cell viability $(\mathrm{P}<0.05$, Fig. 7B), colony formation $(\mathrm{P}<0.05$, Fig. 7C) and proliferation $(\mathrm{P}<0.05$, Fig. 7D) was significantly increased after PTEN downregulation. Furthermore, PTEN knockdown markedly reversed the prevention of cell cycle progression induced by miR-519a suppression $(\mathrm{P}<0.05$, Fig. 7E). Accordingly, PTEN overexpression inhibited cell viability, colony formation, proliferation and promoted G0/G1 phase 


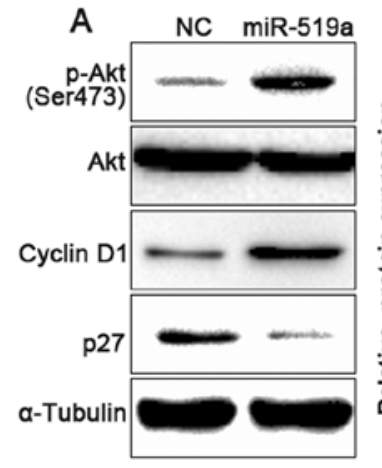

C
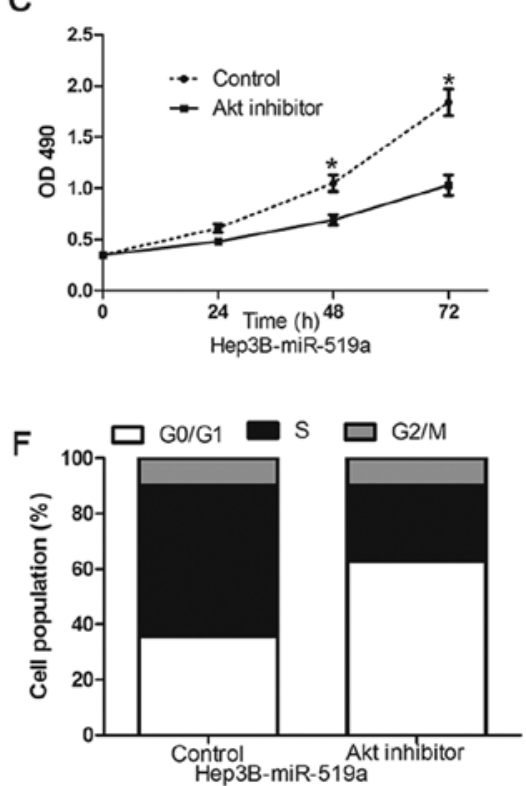

$B$ anti-miR-NC anti-miR-519a
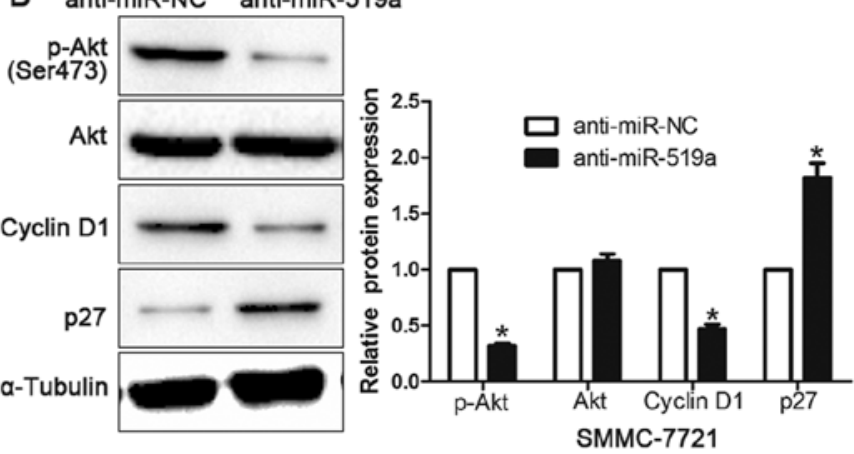

E
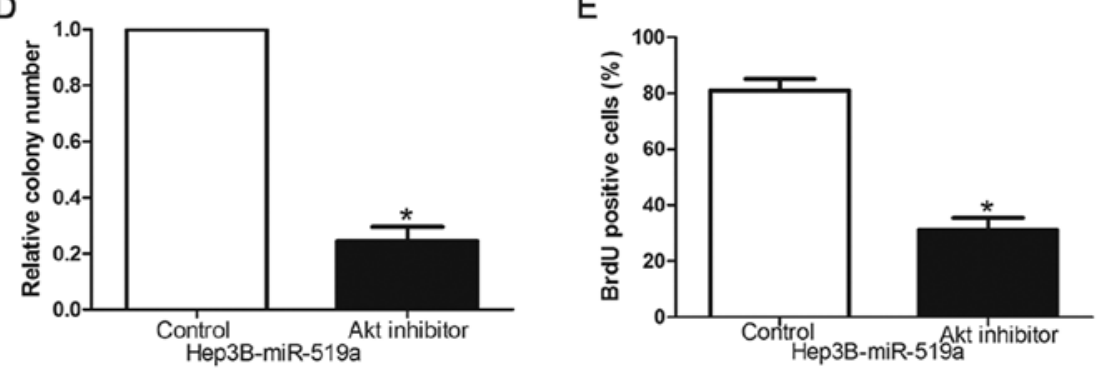

G
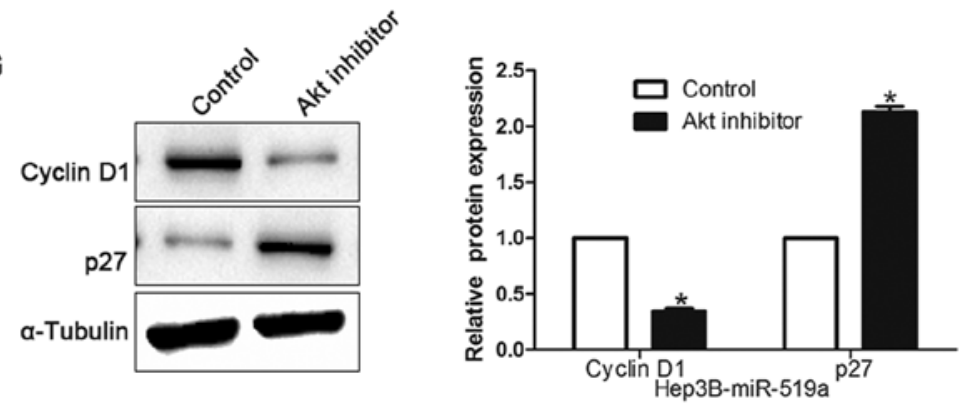

Figure 8. Activation of PI3K/Akt pathway is essential for the biological functions of miR-519a in HCC. (A and B) Western blotting analysis of p-Akt (Ser473), total Akt, Cyclin D1 and p27 protein levels in indicated cells. $\alpha$-tubulin was used as a loading control. Quantification of cell viability (C), colony formation (D), cell proliferation (E) and cell cycle (F) in indicated HCC cells treated with Akt inhibitor $(0.5 \mu \mathrm{M})$. (G) The protein expression level of Cyclin D1 and p27 by western blot analysis in indicated HCC cells treated with Akt inhibitor $(0.5 \mu \mathrm{M})$. Experiments were repeated at least 3 times with similar results, and error bars represent $\pm \mathrm{SD} .{ }^{*} \mathrm{P}<0.05$.

in miR-519a-overexpressing Hep3B cells $(\mathrm{P}<0.05$, Fig. 7A-E, respectively). These data showed that PTEN is a functional downstream mediator of miR-519a in HCC.

PI3K/Akt signaling is essential for the biological function of miR-519a in HCC. Previous studies have demonstrated that PTEN was a negative regulator of PI3K/Akt signaling (19), so we further investigated whether dysregulation miR-519a altered the activity of PI3K/Akt signaling in HCC cells. As shown in Fig. 8A and B, overexpressing miR-519a significantly increased, but silencing miR-519a decreased, the Akt activity and the expression of phosphorylated Akt (Ser473) in HCC cells $(\mathrm{P}<0.05)$. Consistently, the levels of Cyclin D1 and p27, two downstream effectors of PI3K/Akt signaling, were also significantly altered in the miR-519a-deregulated HCC cells $(\mathrm{P}<0.05)$. These results indicate that miR-519a activates PI3K/Akt signaling. Furthermore, we examined whether activation of PI3K/Akt signaling contributed to miR-519a-mediated HCC cell proliferation and cell cycle progression. In Hep3B cells overexpressing miR-519a, inactivation of PI3K/Akt signaling by Akt inhibitor significantly decreased the cell viability $(\mathrm{P}<0.05$, Fig. $8 \mathrm{C})$, colony formation $(\mathrm{P}<0.05$, Fig. $8 \mathrm{D})$, proliferation $(\mathrm{P}<0.05$,
Fig. 8E) and promoted the percentage of $\mathrm{G} 0 / \mathrm{G} 1$ phase $(\mathrm{P}<0.05$, Fig. 8F). Moreover, the Cyclin D1 expression was significantly decreased, but p27 was increased after treating Hep3B cells overexpressing miR-519a with Akt inhibitor ( $\mathrm{P}<0.05$, Fig. $8 \mathrm{G})$. Taken together, our results demonstrate that PI3K/Akt signaling plays an essential function during miR-519a-induced HCC cell proliferation and cell cycle progression.

\section{Discussion}

Increasing evidence demonstrates that aberrant expression or function of miRNAs plays a crucial role in carcinogenesis $(20,21)$. Therefore, identification of novel cancer-related miRNAs and their functional targets may provide promising therapeutic targets for HCC (22). In previous studies, miR519a has been found to promote DNA damage, alter $\mathrm{Ca}^{2+}$ homeostasis and enhance energy production via p21-induced pathways, resulting in growth inhibition of HeLa cells $(16,23)$. Abdelmohsen et al (24) indicated that miR-519 reduced cell proliferation by lowering RNA-binding protein HuR levels in colon cancer. However, Kim et al found that miR-519a was upregulated and correlated significantly with pathological 
grade in ovarian epithelial tumors (14). Moreover, miR-519a promoted cell viability, cell cycle progression and resistance to apoptosis in $\mathrm{ER}^{+}$breast cancer (15).

In the present study, we identified that miR-519a was upregulated in HCC tissues compared to matched tumoradjacent tissues, and this was also confirmed in HCC cells. Elevated expression of miR-519a was significantly correlated with clinicopathological features of HCC, including large tumor size, high histological grade and TNM stage and venous infiltration. In addition, patients with higher miR-519a had a poorer prognosis. Functionally, ectopic expression of miR519a promoted cell viability, colony formation, proliferation and cell cycle progression in HCC cells. Moreover, we identified PTEN as a novel direct target of miR-519a. The functional effects of miR-519a alteration on HCC cells could be reversed by PTEN modulation. Moreover, the expression of PTEN was downregulated in HCC cases and its expression level was inversely correlated with miR-519a. Additionally, we found miR-519a led to upregulation of cell cycle regulator Cyclin D1 and downregulation of p27 through activation of PI3K/Akt pathway. Collectively, our results demonstrate that miR-519a promotes HCC progression through activating Akt signaling pathway by inhibiting PTEN.

Numerous studies showed that activation of the PI3K/Akt signaling pathway was essential to the development and progression of HCC and could modulate the malignant behavior of HCC $(25,26)$, such as cell proliferation, invasiveness, angiogenesis and metastasis $(27,28)$. Of note, PTEN, which acts as an inhibitor of PI3K/Akt pathway, has been found to be inhibited in HCC due to gene deletion or mutation during oncogenesis $(29,30)$. PTEN was an essential regulator of cell proliferation, differentiation, growth and apoptosis, and its deficiency was closely associated with HCC development and progression (31). In addition, PTEN could inhibited tumor cell growth and invasion by suppressing the PI3k/Akt pathway (32). Herein, we discovered that miR-519a activated PI3K/Akt signaling through targeting PTEN, suggesting miR-519a may represent as a potential therapeutic target for HCC treatment.

In conclusion, we demonstrated that miR-519a was frequently overexpressed in HCC tissues and cell lines. miR-519a played a crucial role in the malignant progression of HCC cells through activation of PI3K/Akt by inhibiting PTEN. Therefore, miR-519a has the potential to be a valuable diagnostic and prognostic biomarker and a novel therapeutic target for $\mathrm{HCC}$.

\section{Acknowledgements}

This study was supported by grants from the National Natural Science Foundation of China (no. 81402039) and the Natural Science Basic Research Plan in Shaanxi Province of China (Program no. 2015JM8409).

\section{References}

1. Bosch FX, Ribes J, Díaz M and Cléries R: Primary liver cancer: Worldwide incidence and trends. Gastroenterology 127 (Suppl 1): S5-S16, 2004.

2. El-Serag HB and Rudolph KL: Hepatocellular carcinoma: Epidemiology and molecular carcinogenesis. Gastroenterology 132: 2557-2576, 2007
3. Llovet JM, Di Bisceglie AM, Bruix J, Kramer BS, Lencioni R, Zhu AX, Sherman M, Schwartz M, Lotze M, Talwalkar J, et al; Panel of Experts in HCC-Design Clinical Trials: Design and endpoints of clinical trials in hepatocellular carcinoma. J Natl Cancer Inst 100: 698-711, 2008.

4. Talwalkar JA and Gores GJ: Diagnosis and staging of hepatocellular carcinoma. Gastroenterology 127 (Suppl 1): S126-S132, 2004.

5. Bartel DP: MicroRNAs: Genomics, biogenesis, mechanism, and function. Cell 116: 281-297, 2004.

6. Rosa A and Brivanlou AH: MicroRNAs in early vertebrate development. Cell Cycle 8: 3513-3520, 2009.

7. Vasudevan S, Tong Y and Steitz JA: Switching from repression to activation: microRNAs can up-regulate translation. Science 318: 1931-1934, 2007.

8. Tu K, Zheng X, Dou C, Li C, Yang W, Yao Y and Liu Q: MicroRNA-130b promotes cell aggressiveness by inhibiting peroxisome proliferator-activated receptor gamma in human hepatocellular carcinoma. Int J Mol Sci 15: 20486-20499, 2014.

9. Dou C, Wang Y, Li C, Liu Z, Jia Y, Li Q, Yang W, Yao Y, Liu Q and Tu K: MicroRNA-212 suppresses tumor growth of human hepatocellular carcinoma by targeting FOXA1. Oncotarget 6: 13216-13228, 2015.

10. Chang RM, Yang H, Fang F, Xu JF and Yang LY: MicroRNA331-3p promotes proliferation and metastasis of hepatocellular carcinoma by targeting $\mathrm{PH}$ domain and leucine-rich repeat protein phosphatase. Hepatology 60: 1251-1263, 2014.

11. Su H, Yang JR, Xu T, Huang J, Xu L, Yuan Y and Zhuang SM: MicroRNA-101, down-regulated in hepatocellular carcinoma, promotes apoptosis and suppresses tumorigenicity. Cancer Res 69: 1135-1142, 2009.

12. Yang X, Zhang XF, Lu X, Jia HL, Liang L, Dong QZ, Ye QH and Qin LX: MicroRNA-26a suppresses angiogenesis in human hepatocellular carcinoma by targeting hepatocyte growth factorcMet pathway. Hepatology 59: 1874-1885, 2014.

13. Santhekadur PK, Das SK, Gredler R, Chen D, Srivastava J, Robertson C, Baldwin AS Jr, Fisher PB and Sarkar D: Multifunction protein staphylococcal nuclease domain containing 1 (SND1) promotes tumor angiogenesis in human hepatocellular carcinoma through novel pathway that involves nuclear factor $\kappa \mathrm{B}$ and miR-221. J Biol Chem 287: 13952-13958, 2012.

14. Kim TH, Kim YK, Kwon Y, Heo JH, Kang H, Kim G and An HJ: Deregulation of miR-519a, 153, and 485-5p and its clinicopathological relevance in ovarian epithelial tumours. Histopathology 57: 734-743, 2010

15. Ward A, Shukla K, Balwierz A, Soons Z, König R, Sahin O and Wiemann S: MicroRNA-519a is a novel oncomir conferring tamoxifen resistance by targeting a network of tumour-suppressor genes in ER+ breast cancer. J Pathol 233: 368-379, 2014.

16. Abdelmohsen K, Srikantan S, Tominaga K, Kang MJ, Yaniv Y, Martindale JL, Yang X, Park SS, Becker KG, Subramanian M, et al: Growth inhibition by miR-519 via multiple p21-inducing pathways. Mol Cell Biol 32: 2530-2548, 2012.

17. Abdelmohsen K, Srikantan S, Kuwano Y and Gorospe M: miR-519 reduces cell proliferation by lowering RNA-binding protein HuR levels. Proc Natl Acad Sci USA 105: 20297-20302, 2008.

18. Yang W, Dou C, Wang Y, Jia Y, Li C, Zheng X and Tu K: MicroRNA-92a contributes to tumor growth of human hepatocellular carcinoma by targeting FBXW7. Oncol Rep 34: 2576-2584, 2015.

19. Jiang L, Wang C, Lei F, Zhang L, Zhang X, Liu A, Wu G, Zhu J and Song L: miR-93 promotes cell proliferation in gliomas through activation of PI3K/Akt signaling pathway. Oncotarget 6: 8286-8299, 2015.

20. Garzon R, Calin GA and Croce CM: MicroRNAs in Cancer Annu Rev Med 60: 167-179, 2009.

21. Croce CM and Calin GA: miRNAs, cancer, and stem cell division. Cell 122: 6-7, 2005.

22. Lu J, Getz G, Miska EA, Alvarez-Saavedra E, Lamb J, Peck D, Sweet-Cordero A, Ebert BL, Mak RH, Ferrando AA, et al: MicroRNA expression profiles classify human cancers. Nature 435: 834-838, 2005.

23. Marasa BS, Srikantan S, Martindale JL, Kim MM, Lee EK, Gorospe $\mathrm{M}$ and Abdelmohsen K: MicroRNA profiling in human diploid fibroblasts uncovers miR-519 role in replicative senescence. Aging (Albany NY) 2: 333-343, 2010.

24. Abdelmohsen K, Kim MM, Srikantan S, Mercken EM, Brennan SE, Wilson GM, Cabo R and Gorospe M: miR-519 suppresses tumor growth by reducing HuR levels. Cell Cycle 9: $1354-1359,2010$ 
25. Courtney KD, Corcoran RB and Engelman JA: The PI3K pathway as drug target in human cancer. J Clin Oncol 28: 1075-1083, 2010.

26. Fresno Vara JA, Casado E, de Castro J, Cejas P, Belda-Iniesta C and González-Barón M: PI3K/Akt signalling pathway and cancer. Cancer Treat Rev 30: 193-204, 2004.

27. Cheng GZ, Park S, Shu S, He L, Kong W, Zhang W, Yuan Z, Wang LH and Cheng JQ: Advances of AKT pathway in human oncogenesis and as a target for anti-cancer drug discovery. Curr Cancer Drug Targets 8: 2-6, 2008.

28. Cappellini A, Tabellini G, Zweyer M, Bortul R, Tazzari PL, Billi AM, Falà F, Cocco L and Martelli AM: The phosphoinositide 3-kinase/Akt pathway regulates cell cycle progression of HL60 human leukemia cells through cytoplasmic relocalization of the cyclin-dependent kinase inhibitor p27(Kip1) and control of cyclin D1 expression. Leukemia 17: 2157-2167, 2003.
29. Song MS, Salmena L and Pandolfi PP: The functions and regulation of the PTEN tumour suppressor. Nat Rev Mol Cell Biol 13: 283-296, 2012.

30. Cantley LC and Neel BG: New insights into tumor suppression: PTEN suppresses tumor formation by restraining the phosphoinositide 3-kinase/AKT pathway. Proc Natl Acad Sci USA 96: 4240-4245, 1999.

31. Rahman MA, Kyriazanos ID, Ono T, Yamanoi A, Kohno H, Tsuchiya $\mathrm{M}$ and Nagasue $\mathrm{N}$ : Impact of PTEN expression on the outcome of hepatitis $\mathrm{C}$ virus-positive cirrhotic hepatocellular carcinoma patients: Possible relationship with COX II and inducible nitric oxide synthase. Int J Cancer 100: 152-157, 2002.

32. Tamura M, Gu J, Tran $\mathrm{H}$ and Yamada KM: PTEN gene and integrin signaling in cancer. J Natl Cancer Inst 91: 1820-1828, 1999. 